\title{
HEILIGENKREUZ 217 UND DIE ANFÄNGE DER SCHRIFTKULTUR IN BÖHMEN UM 1000: DIE PRAGER KIRCHE IM ZEITALTER BISCHOFS THIDDAG*
}

\author{
DALIBOR HAVEL \\ Philosophische Fakultät, Masaryk-Universität \\ E-mail: havel@phil.muni.cz \\ DAVID KALHOUS \\ Philosophische Fakultät, Masaryk-Universität \\ E-mail:12320@mail.muni.cz
}

\begin{abstract}
Heiligenkreuz 217 and the Beginnings of the Written Culture in Bohemia round 1000: The Church of Prague in the Age of Bishop Thiddag

The codicological and paleographical analysis of the ms. Heiligenkreuz, Stiftsbibliothek Nr. 217 combined with the scrutiny of the content introduces the audience into the material evidence for the begginings of the literacy in Bohemia at the end of the 10th century. It demonstrates that the above mentioned codex, which mostly included poenitentials and Carolingian capitularies, was written ca. 950 in today's Southern Germany. Before 1000, probably c. 990, it was donated to or bought for Prague bishopric (founded 976), where it was not just used and read, but the churchmen also copied in it documents important for Bohemian Church (letter of Stephan V, 885 sent to Moravia), or diverse texts compiled in Prague (contract between St. Adlabert-Vojtěch, bishop of Prague, duke Boleslav II and the elites; two sermons probably written by St. Adalbert).

Keywords: manuscript; St. Adalbert; bishopric Prague; Heiligenkreuz
\end{abstract}

In unserem Beitrag möchten wir eine für die Lösung der besagten Fragen sehr wichtige Quelle präsentieren, und zwar die Pergamenthandschrift Nr. 217 der Stiftsbibliothek Heiligenkreuz ${ }^{1}$. Es handelt sich um eine umfangreiche Sammelhandschrift ${ }^{2}$, die ein bisschen simplifiziert gesagt canones poenitentiales an einer und eine Sammlung von verschiedenen Capitularia und Leges an anderer Seite enthält. Diese Handschrift wurde von

* Paläographische und kodikologische Analyse wurde von D. Havel durchgeführt; Inhalt analysierte D. Kalhous. Die Analysen wurden durch das Marie-Curie Stipendium, FWF M2120-G28 und MUNI/A/1175/2018 finanziert. Tiefere Analyse der Technik der Compillierung des Inhalts ist in Vorbereitung.

1 Benedict GSELL, Verzeichniss der Handschriften in der Bibliothek des Stiftes Heiligenkreuz (Handschriften-Verzeichnisse. Bd. 1). Wien 1891, S. 174 f. (Nr. 217). Zum Skriptorium und Bibliothek in Heiligenkreuz vgl. Alois HAIDINGER - Franz LACKNER, Die Bibliothek und das Skriptorium des Stiftes Heiligenkreuz unter Abt Gottschalk (1134/1147) (Codices manuscripti et impressi. Zeitschrift für Buchgeschichte - Supplementum 11). Purkersdorf 2015 - der Heiligenkreuz 217 wurde im 12. Jhdt. in Heiligenkreuz noch nicht aufbewahrt.

2 Zum Inhalt vgl. Hubert MORDEK, Bibliotheca capitularium regum Francorum manuscripta: Überlieferung und Traditionszusammenhang der fränkischen Herrschererlasse. München 1995, S. 158-172. 
Wilhelm Wattenbach in den 40en Jahren des 19. Jahrhunderts ausführlich studiert ${ }^{3}$, und Wattenbach war der erste, der auch zwei neuere Zusätze bemerkt hatte, die man sicher in Böhmen in diese Handschrift eingetragen musste: es handelte sich um von Wattenbach edierte das so genannte Edictum Boleslai ducis vom Jahre $992^{4}$ und eine Abschrift des Apostelbriefs vom Stephan V. an mährischen Herzog Svatopluk aus dem Jahre etwa $885^{5}$.

Lange nach Wattenbach wurde ein neues Bohemicum in dieser Handschrift entdeckt, und zwar die sogenannte Ammonitio et exhortatio episcopalis de ammonicione ad presbyteros, eine bischöfliche Rede an Klerus, die mit dem zweiten Prager Bischof Vojtěch/ Adalbert verbindet und als eine neue Entdeckung von Dušan Třšstík und Jana Zachová im Jahre 2001 publiziert und ediert wurde ${ }^{6}$.

Eine ausführlichere paläographische und speziell kodikologische Analyse aber bis jetzt fehlte. Und so wurde ein Studium in der Stiftsbibliothek in Heiligenkreuz realisiert ${ }^{7}$, und in unserer Studie beabsichtigen wir mit den Grundlinien dieses Studiums der Originalhandschrift Heiligenkreuz 217 und mit seinen Hauptergebnissen bekannt machen.

Cod. 217 enthält 330 Pergamentfolia, heute in 44 Lagen eingeteilt, die Lagen überwiegend von Quaternionen und Ternionen geschaffen wurden. Diese Handschrift wurde sehr schnell, man würde sagen übereilt, in einem bayerischen Skriptorium im 10. Jahrhundert geschrieben. Das bezeugt die paläographische Analyse, die gezeigt hat, dass die Schreiberarbeit der einzelnen Hände im Prinzip einzelnen Lagen entsprechend geteilt wurde. Und so viele Blätter besonders am Ende der Lagen ursprünglich leer geblieben hatten. Dieser günstigere Umstand nutzte der Schreiber des ältesten böhmischen Eintrags in dieser Handschrift aus: auf der recto-Seite des Folios 76 befindet sich das schon erwähnte Edictum Boleslai ducis, das in Form von Traditionsnotiz eine öffentliche Erklärung vom Herzog Boleslav II. über die Vereinbarungen mit dem Bischof Adalbertus aus dem Jahre 992 aufzeichnet. Aus den paläographischen Gründen können wir sagen, dass dieser Eintrag schon kurz nach benachrichtigtem Geschehnis, also noch am Ende des 10. Jahrhunderts geschrieben wurde ${ }^{8}$. Und schon damals musste die Handschrift 217 sicher in Böhmen, in Prag aufbewahrt werden. Es handelt sich um das älteste bis jetzt bekannte und in Böhmen geschriebene lateinische Text. Die Veröffentlichung dieses "Ediktes“ im ersten Teil des Böhmischen Diplomatars von Gustav Friedrich bewirkte leider eine Verwirrung: Friedrich interpretierte es als eine Abschrift des 12. Jahrhun-

3 Wilhelm WATTENBACH, Beiträge zur Geschichte der christlichen Kirche in Maehren und Boehmen. Wien 1849 und DERS., Reise nach Osterreich in den Jahren 1847, 1848, 1849, in: Archiv der Gesellschaft für ältere deutsche Geschichtskunde 10 (1851), S. 597 f. Dazu vgl. Franz ZAGIBA, Der Kodex 217 der Stiftsbibliothek Heiligenkreuz in Niederosterreich als Quelle zur Kirchengeschichte Böhmens und Mährens, in: Millenium dioeceseos Pragensis 973-1973: Beiträge zur Kirchengeschichte Mitteleuropas im 9.-11. Jahrhundert. Wien/Köln/Graz 1974, S. 66.

4 Codex diplomaticus et epistolarius Regni Bohemiae. Bd. 1, hg. von Gustav FRIEDRICH. Praha 1907, S. 43 (Nr. 37).

5 Ebd., S. 22-26 (Nr. 26), bzw. Magnae Moraviae Fontes Historici. Bd. 3, hg. von Dagmar BARTOŇKOVÁ - Lubomír HAVLÍK - Jaroslav LUDVÍKOVSKÝ - Radoslav VEČERKA. Brno 1969, S. 179-190 (Nr. 101).

6 Jana ZACHOVÁ - Dušan TŘEŠTÍK, Adhortace De ammonicione ad presbiteros a biskup Vojtěch, in: ČČH 99 (2001), S. 279-293.

7 Neuere paläographisch-kodikologische Analyse bei Dalibor HAVEL, Počátky latinské písemné kultury $v$ českých zemích: nejstarší latinské rukopisy a zlomky v Čechách a na Moravě. Brno 2018, S. 79-95.

8 HAVEL, Počátky latinské písemné kultury, S. 91, Abb I. 
derts $^{9}$ und dieser Standpunkt beeinflusste die anderen Meinungen, vornehmlich in der tschechischen Literatur ${ }^{10}$. Diese Meinung ist aber von den paläographischen Gründen auszuschließen. Tatsächlich liegt vor uns eine der ältesten Schichten von Memoria, die bei der Prager Kirche in die Handschrift Heiligenkreuz 217 eingetragen wurde.

$\mathrm{Zu}$ etwa jüngerer Schicht dieser Memoria gehört die Ammonitio des Prager Bischofs Adalbert. Sie wurde von zwei wieder verschiedenen Händen geschrieben ${ }^{11}$, die aber im Vergleich mit der Schreiberhand des Edictums Boleslai keine morphologische Verbindungen aufweisen; d. h. dass wir noch um 1000 über kein Skriptorium, keine systematische, kontinuierliche und organisierte Schreibertätigkeit in der Umgebung des Prager Kapitels sprechen können. Die Ammonitio füllt zwei letzte Folia der siebten Lage (Fol. 51 und 52) und die obere Hälfte des ersten Folios der folgenden, achten Lage aus. Um den Text von Ammonitio zu beenden, war es nötig, den Anfang des ursprünglichen Textes auf der recto-Seite des Folios 53 zu radieren - dadurch hier ein Palimpsest entstand. Die letzten sechs Folia dieser Lage wurden ausgeschnitten und sind heute verloren.

Die interessanteste Situation kommt in Zusammenhang mit dem letzten Bohemicum vor, das in die Handschrift 217 eingetragen wurde, und zwar mit der Abschrift vom Apostelbrief des Papstes Stephanus an Svatopluk. Anfang der Abschrift beginnt typisch am Ende der Lage, in diesem Fall, am Ende der Lage „I“ (vom Folio 5verso bis Folio 6verso), aber der leere Platz war nicht genügend, um den ganzen Text aufzunehmen. So wurde eine ganz neue Lage geschaffen, die aus zwei Doppelblätter (Fol. 78-79 und Fol. 80-81) und einem Einzelblatt (Fol. 77) bestand. Diese neue Lage wurde noch im 12. Jahrhundert mit der Kustode „XII“ bezeichnet. Während die Vollendung des Stephanus-Brief sich auf den Folia 78 bis 81 befindet, Fol. 77 ein bis jetzt ungedruckten Sermon mit Inzipit Quia semel vestrę caritatis enthält, das wir als ein neues Adalbertus-Werk interpretieren müssen ${ }^{12}$.

Es ist sicher keine größere Überraschung, dass auch diese Texte (Apostelbrief und Sermon) von anderen verschiedenen Händen geschrieben wurden ${ }^{13}$.

Wie oben bewiesen wurde, ist nicht nur am Umstand zu zweifeln, dass die Handschrift Heiligenkreuz 217 schon seit 990/1000 in Prag aufbewahrt wurde, sondern darüber hinaus auch um vier weitere Texte von unterschiedlichem Charakter erweitert wurde. An dieser Stelle interessiert uns die inhaltliche Perspektive der besagten Handschrift. Wir als Historiker neigen oft dazu, das kulturelle Niveau gewisser Epochen oder Regionen aufgrund von Texten zu bewerten, die zu einer bestimmten Zeit oder in einem definierten Raum verfasst worden sind. Das ist allerdings irreführend, wenn nicht sogar ganz falsch. Insbesondere für das Mittelalter ist diese Perspektive unzutreffend, da die Zeitgenossen mit textueller Originalität und Autorschaft ganz anders gearbeitet haben als wir es heute tun ${ }^{14}$. Viele Texte weisen einen langen Überlieferungsweg auf und haben Autoren

9 Codex diplomaticus et epistolarius regni Bohemiae. Bd. 1, S. 43 (Nr. 37: Ex codice ms. saec. XII monasterii s. Crucis nemorosa).

10 Z. B. Rostislav NOVÝ- Jiř́ SLÁMA, Slavníkovci ve středověkém pisemnictví. Praha 1987, S. 358 ff.

11 ZACHOVÁ - TŘEŠTÍK, Adhortace De ammonicione, S. 281, Anm. 16.

12 Die Edition dieses Sermons wird in einer selbstständigen Studie vorbereitet.

13 Erste 12 Lagen der Hs. Heiligenkreuz 217 in Übersicht vgl. auf dem „Tafel 1“ am Ende dieses Beitrags.

14 Zuletzt heftig kritisiert von Maximilian DIESENBERGER, Der Cvp 420 - die Gemeinschaft der Heiligen und ihre Gestaltung im frühmittelalterlichen Bayern, in: Monique Goullet - Martin Heinzelmann - Christiane Veyrard-Cosme (Hgg.), L’hagiographie mérovingienne à travers ses réécritures. 
auch in ferneren Regionen Europas für mehrere Jahrhunderte beeinflusst. Das ist auch bei vielen normativen karolingischen Texten der Fall, die in unserer Handschrift zu lesen sind. Wir werden jetzt die Handschrift aus inhaltlicher Perspektive analysieren, um das kulturelle Niveau in Prag um 1000 zu dokumentieren. Indirekt sind die Texte auch ein Spiegel der zeitgenössischen Gedankenwelt des hohen Klerus im Reich, und darüber hinaus stellen sie eine Quelle dar, die zum besseren Verständnis des „Reformprogramms“ von Thiddags bekannterem Vorgänger, dem Hl. Adalbert, hilfreich sein kann.

Wenn wir von den in Prag verfassten Texten einmal absehen, können wir die in Heiligenkreuz 217 überlieferten Texte nach inhaltlichen Kriterien in zwei Gruppen unterteilen. Eine Gruppe besteht aus den normativen Texten, unter denen wir noch vier Untergruppen unterscheiden können: erstens handelt es sich dabei um karolingische Kapitularien, die entweder selbständig, oder im Rahmen der Ansegis-Sammlung überliefert sind ${ }^{15}$; zweitens, sind in der Handschrift die zwei süddeutschen Leges, die Lex Baiuwariorum und Lex Alamannorum, zu finden ${ }^{16}$; drittens beinhaltet die Hs. Synodalbeschlüsse, allen voran diejenigen der Synode in Worms (868), und andere kanonistische Quellen ${ }^{17}$; viertens findet man darin außerdem ein Fragment des bischöflichen Kapitulars von Gherbald von Lüttich (Kap. 7, 8, 12) ${ }^{18}$. An den restlichen Folien der Handschrift sind Buß- und Beichtordnungen anzutreffen ${ }^{19}$.

Unsere Handschrift ist keineswegs isoliert entstanden, sondern ist mit zwei weiteren Handschriften verwandt, die heute in Paris (P, BNF lat. 3878) und in München ( $\mathrm{M}, \mathrm{Clm}$ 3853) aufbewahrt werden, wobei ihre Schriftheimat nach Süddeutschland des 10. Jahrhunderts situiert werden darf - höchstwahrscheinlich in Brixen und in Augsburg. Alle sind höchstwahrscheinlich mit der Absicht kompiliert, um dem Bischof als Handbuch zu dienen ${ }^{20}$. Die beste Variante bietet die Münchener Handschrift, die als Quelle der nicht mehr existenten Handschrift X diente ${ }^{21}$. Unabhängig voneinander haben die Schreiber von $\mathrm{P}$ und $\mathrm{H}$ diese nicht mehr existierende Handschrift als Vorlage benutzt, wobei der Schreiber von P mit der Hs. X mit viel größerer Vorsicht und Akribie arbeitete als die Schreiber von $\mathrm{H}^{22}$. Dieser Sichtweise entsprechen auch die Ergebnisse der paläographischen und kodikologischen Analysen, welche bestätigen, dass die Hs. überaus rasch verfasst worden sein musste. Gewisse inhaltliche Parallelen zu unserer Handschriftengruppe

Ostfildern 2010, S. 219-248; damit ist z. B. die Perspektive der MGH SRM irreführend (Heiligen, die damals gelebt haben, dessen Legenden aber auch später verfasst worden sind). Vergl. auch Gert MELVILLE, Spätmittelalterliche Geschichtskompendien: eine Aufgabenstellung, in: Römische historische Mitteilungen 22 (1980), S. 51-104, hier zum Kompilationsprinzipien bes. S. 61-77.

15 Heiligenkreuz 217, Ff. 203v-330v.

16 Heiligenkreuz 217, Ff. 183r-203v.

17 Heiligenkreuz 217, Ff. 106r-183r. Siehe zu den Synodalbeschlüssen von Worms und ihrer Überlieferung bes. Wilfried HARTMANN, Das Konzil von Worms 868: Überlieferung und Bedeutung. Göttingen 1977.

18 Heiligenkreuz 217, F. 279v.

19 Heiligenkreuz 217, Ff. 30v-105v. Vergl. Raymund KOTTJE, Die Bussbücher Halitgars von Cambrai und des Hrabanus Maurus: ihre Überlieferung und ihre Quellen. Berlin/New York 1980.

20 Vergl. auch John ELDEVIK, Episcopal Power and Ecclesiastical Reform in the German Empire: Tithes, Lordship, and Community, 950-1150. Cambridge 2012, S. 68.

21 Silke HANSEN, Rechtscorpora in den Handschriften CLM 3853, Heiligenkreuz 217 und Par. Lat. 3878. Tübingen 1992 (ungedruckte Magisterarbeit), S. 71-93, hier bes. S. 90.

22 Ebd., S. 80. 
lassen sich auch noch in einer Handschrift Vaticanus lat. 5751 finden, die ebenfalls während des 10. Jahrhunderts in Oberitalien geschrieben wurde ${ }^{23}$.

Einen hohen Stellenwert nimmt in Heiligenkreuz 217 die Lex Alamannorum ein. Auf den ersten Blick könnte das zwar in solchen bischöflichen „Handbuch“ überraschen, aber dieses Gesetzbuch war im frühmittelalterlichen Europa mit seinen 55 Handschriften weit verbreitet. ${ }^{24}$ Bis 1000 kommt diese Lex in der Regel nicht in selbständigen Handschriften vor, sondern in Sammelhandschriften, wo sie mit weiteren Leges barbarorum abgeschrieben wurde. Man kann dieses Phänomen und die reiche Überlieferung der Lex Alamannorum mit der Personalität des Rechts und mit dem Bedarf an Rechtstexten erklären, die ermöglichten, die Streitigkeiten innerhalb der multiethnischen Gesellschaft des fränkischen Reiches zu schlichten. Obwohl diese Annahme überzeugend klingt und unseren Kenntnissen nicht widerspricht, finden wir sie nicht korrekt. Es ging vielmehr um eine Sammlung der Rechtssprüche, die dem Rechtskenner eine Anleitung zur Lösung gewisser Situationen gaben, aber keine bindende Kraft hatten. Diese Funktion besaß die Lex Alamannorum vielleicht auch in Prag $^{25}$. Für das Milieu des Prager Klerus war dieses Gesetzbuch umso geeigneter, weil darin der Kirche und ihrem Schutz besondere Aufmerksamkeit geschenkt wird. Übrigens wurde sie wohl in ähnlicher Weise wie die Lex Baiuwariorum in kirchlichen Kreisen verfasst. Wir sind allerdings nicht imstande, ihre direkte Auswirkung in den böhmischen Ländern zu bezeugen.

Die Kapitulariensammlung des Ansegis gehört auch zu den am häufigsten überlieferten frühmittelalterlichen Texten - heute kennen wir sie anhand von mehr als 60 Handschriften ${ }^{26}$. Die Sammlung wurde schon bald nach ihrer Kompilierung verbreitet und war durchaus beliebt, worüber fast 30 Handschriften aus dem 9. Jh. zeugen. Mehr als 20 Handschriften kennen wir noch aus dem folgenden Jahrhundert, später wurde sie nur selten abgeschrieben. Die meisten Handschriften sind im Westfrankenreich entstanden ${ }^{27}$. Ihre Popularität bestätigt schon Hinkmar von Rheims, der nicht nur diese Sammlung ausschließlich benutzte, wenn er sich auf die Kapitularien Karls des Großen und Ludwigs des Frommen bezog, sondern auch selbst in seiner Bibliothek sechs ihrer Handschriften besaß. Ihre Auswirkung in den böhmischen Ländern deutet lediglich auf ein wichtiges Indiz hin: das Homiliar von Opatowitz, eine Sammlung, die auch ein Poenitential beinhaltet und um 1100 in Prag kompiliert worden ist, wiederholt Wort für Wort eine Regelung, die die Pfarrzugehörigkeit bespricht ${ }^{28}$.

${ }^{23}$ Ebd., S. 102-110.

${ }^{24}$ Leges Alamannorum (MGH Leges nationum Germanicarum. Bd. 5. Teil. 1.), hg. von Karl August ECKHARDT. Hannover 1966, S. 12-18.

25 Zum Widerhall der Lex Salica in Böhmen in Legenda Christiani vgl. Petr SOMMER, Začátky křestanství v Čechách: kapitoly z dějin raně středověké duchovní kultury. Praha 2001, S. 104 ff., 129 f.; DERS., Smrt kněžny Ludmily a začátky české sakrální architektury, in: ČČH 98 (2000), S. 229-260.

26 Die Kapitulariensammlung des Ansegis (MGH Capitularia regum Francorum. Nova. Series. Bd. 1), hg. von Gerhard SCHMITZ. Hannover 1996, S. 71-191.

27 Vgl. Anm. 11, und auch Lotte KÉRY, Canonical collections of the Early Middle Ages, ca. 400-1140: a Biographical Guide to the Manuscripts and Literature. Washington, DC 1999, S. 92-100; Wilfried HARTMANN, Kirche und Kirchenrecht um 900: die Bedeutung der spätkarolingischen Zeit für Tradition und Innovation im kirchlichen Recht. Hannover 2008, S. 90-93.

28 Die Kapitulariensammlung des Ansegis, S. 511 (I \$ 147): „DE PARROCHIANIS ALTERIUS PRESBITERI. Ut nullus presbiter alterius parrochianum, nisi in itinere fuerit vel placitum ibi habuerit, ad missam recipiat."; Das Homiliar des Bischofs von Prag (Beiträge zur Geschichte Böhmens, Abt. 1. 
Ein weiteres wichtiges Zeugnis dafür, dass die Hs. in Prag benutzt worden ist, bietet die Hs. selbst. Sie beinhaltet einen Vertrag, der zwischen dem Hl. Adalbert, Boleslav II. und Mächtigen des Landes abgeschlossen worden ist. Adalbert verlangte damals u. a. das Recht, Ehen aufzulösen, die zwischen den Verwandten entgegen dem kanonischen Recht geschlossen worden waren ${ }^{29}$. In dieser Angelegenheit hat sich der Bischof von Prag von den Synodalbeschlüssen aus Worms inspirieren lassen ${ }^{30}$, die ein entscheidender Teil unserer Hs. sind. Auch zwei weitere Texte in Heiligenkreuz 217, nämlich eine Exhortatio und eine weitere, an die Laien gerichtete Predigt, illustrieren, dass Adalbert von Prag ein fleißiger Leser der karolingischen Texte war. Ein Indiz dafür, dass hl. Adalbert auch Poenitential von Hrabanus Maurus benutzt hat, kann eine Warnung den Priestern sein, dass sie keine Autorität mehr haben werden, falls sie selbst die Regeln seines Standes ignorieren werden ${ }^{31}$.

Man spricht im obengenannten Vertrag auch über den Zehnten, der eingesammelt werden sollten ${ }^{32}$ und die Adalbert gemäß der Legenden in vier Teile aufteilte ${ }^{33}$. Hier gilt es auf die karolingischen Texte zu verweisen, die zwar in Heiligenkreuz 217 überliefert sind ${ }^{34}$, die wir aber nicht direkt textkritisch vergleichen können, da unsere Informatio-

Bd. 1), hg. von Ferdinand HECHT. Praha 1863, S. 22: „Nullus alterius parrochianum, nisi in itinere, vel sibi placitum fuerit ad missam recipiat."

29 Codex diplomaticus et epistolarius regni Bohemiae. Bd. 1, S. 43 (Nr. 37): „... separare ea coniugia, que infra parentelam contra sacram legem coniuncta esse reperirentur." Für streitende Autoritäten der Karolingerzeit vgl. Karl UBL, Inzestverbot und Gesetzgebung: die Konstruktion eines Verbrechens (300-1100). Berlin/New York 2008, S. 294-307; Mayke de JONG, To the Limits of Kinship: AntiIncest Legislation in the Early Medieval West (500-900), in: Jan Bremmmer (Hg.), From Sappho to de Sade: Moments in the History of Sexuality. London 1989, S. 36-59.

30 Capitula synodi Wormaciensis, in: MGH LL Concilia. Bd. 4: Die Konzilien der karolingischen Teilreiche 860-874, hg. von Wilfried HARTMANN. Hannover 1998, S. 269 (Kap. 14) und S. 272 (Kap. 19).

31 Adalbert von Prag (?), Ammonicio et exortacio episcopalis, f. 52r: „Qua fiducia gregem, cui vos preesse divina clemencia voluit, de iniusta secundarum subintroductione uxorum, ipsi vestras contra regulam possidentes, commonere temptemini?". Vgl. Hrabanus Maurus, Poenitentiale ad Heribaldum, Kap. 12, in: Heiligenkreuz 217, f. 82r.

32 Codex diplomaticus et epistolarius Regni Bohemiae. Bd. 1, S. 43 (Nr. 37: „... decimas congregandi licentiam dedit.“). Vgl. Die Kapitulariensammlung des Ansegis, S. 558 (II § 37: „Qui vero decimas post crebras admonitiones et praedicationes sacerdotum dare neglexerint, excommunicentur.")

33 Cf. S. Adalberti Pragensis episcopi et martyris Vita prior, hg. von Jadwiga KARWASINSSKA, in: Monumenta Poloniae Historica. Series nova. Bd. 4. Teil 1. Warszawa 1962, S. 14-19 (Kap. 9-12); Bruno von Querfurt, Sancti Adalberti Vita altera, hg. von Jadwiga KARWASIŃSKA, in: Monumenta Poloniae Historica. Series nova. Bd. 4. Teil 2. Warszawa 1969, S. 10-13 (Kap. 11). Siehe auch Arnonis Instructio pastoralis, „Instructio pastoralis“, in: Albert WERMIGHOFF (Hg.), MGH LL Concilia. Bd. 2: Concilia aevi Karolini. Teil 1/1: 742-817. Hannover/Leipzig 1906, S. 200 (Kap. 10), oder die letzte Edition von Raymond ETAIX, Un manuel de Pastorale de l’epoque carolingienne, in: Revue Bénédictine 91 (1981), S. 105-133, hier S. 120. Weitere Synodalsermo wurde von Rudolf POKORNY, Ein unbekannter Synodalsermo Arns von Salzburg, in: Deutsches Archiv 39 (1983), S. 379-394 identifiziert und ähnliche Anweisungen verfasste auch Hinkmar von Reims, cf. Hinkmar von Rheims, Quae exsequi debeat episcopus, et qua cura tueri res et facultates ecclesiasticas, in: Jacques-Paul MIGNE (Hg.), Patrologia Latina. Bd. 125. Paris 1852, col. 1087-1094. Das heißt, dass das Modelbischof nicht mit konkretem Genre verknüpft wurde, sondern die Grenzen der Genre überquerte. Für Bistumsverwaltung in Spätkarolingerzeit vgl. Martina STRATMANN, Hinkmar von Reims als Verwalter von Bistum und Kirchenprovinz. Sigmaringen 1991.

34 Die Kapitulariensammlung des Ansegis (I \$153): „Ut decimae in potestate episcopi sint, qualiter a presbiteris dispensentur; Gherbald of Liège, Erstes Kapitular, hg. von Peter BROMMER. MGH Capitularia Episcoporum. Bd. 1. Hannover 1984, S. 17 f. (\$ 5: „Ut ipsi sacerdotes populi suscipiunt decimas et nomina eorum, quicumque dederint, scripta habeant et secundum auctoritatem canonicam coram testibus dividant et ad ornamentum ecclesieae primam elegant partem, secundam autem ad usum 
nen nicht aus Adalbert's dossier schöpfen, sondern wir hier über Adalberts Tätigkeit nur durch die Legenden benachrichtigt sind, die aber nicht in Prag, sondern in Lüttich und in Italien verfasst worden sind. Das gilt auch für weitere Punkte, die der Hl. Adalbert angeblich kritisierte - nämlich Priesterehen, den Verkauf von christlichen Sklaven an Juden ${ }^{35}$ sowie Kirchenfeste. Hinsichtlich dessen ist uns auch nur das bekannt, was uns die Hagiographen mitteilen. Diese stilisierten den Hl. Adalbert allerdings als idealen Bischof hoch. Deshalb müssen wir die hagiographischen Schilderungen seiner Konflikte mit den Přemysliden und mit den böhmischen Eliten mit Vorsicht beurteilen - mindestens einige seiner Schritte führten nicht nur zur Festigung des Prager Bistums, sondern auch der frühen Monarchie.

Den Zeitraum des dritten Prager Bischoffs Thiddag, des Nachfolgers des hl. Adalberts, bringt man mit der ersten Erwähnung der Prager Kapitelschule gewöhnlich in Zusammenhang. Es handelt sich um die bekannte, den Mag. Hubaldus betreffende Bemerkung der Gesta episcoporum Leodicensium von Anselm de Liége; der in Prag „nonnulla ... christianae religionis documenta dedisset", und zwar im Zeitraum des Lütticher Bischofs Baldrich II. (1008-1018) .

Falls in Prag wirklich schon an der Wende vom 10. zu 11. Jahrhundert ein intellektuelles Zentrum wäre, das eine solche Persönlichkeit wie Mag. Hubaldus anziehen könnte, müssen wir auch voraussetzen, dass man hier auch andere Symptome des intellektuellen Lebens verfolgen solle.

In der ersten Linie haben wir die bescheidenen Spuren der lateinischen Schriftstücke im Sinne, die wir mit der Prager Kirche verbinden können und die im betreffenden Zeitraum abgeschrieben wurden. Obwohl unsere Analysen gezeigt haben, dass schon am Ende des 10. Jahrhunderts in Prag St. Adalbert/Vojtěch stark in dem zeitgenössischen kulturellen und kirchlichen Milieu verwurzelt war, das sowohl das Auswahl der Regeln, als auch die Heftigkeit, mit der er diese Regeln durchgesetzt habe, reihen ihn zur Avantgarde des damaligen Reichsklerus ${ }^{37}$. Die Handschrift Heiligenkreuz 217 ist hier ein der wichtigsten Zeugen.

pauperum, atque peregrinorum per eorum manus misericorditer cum omni humilitate dispensant, tertiam vero partem sibimetipsis solis sacerdotes reservant.").

35 Dazu auch Hrabanus in seinem Brief an Chorbischof Reginbald on Metz, siehe Heiligenkreuz 217, f. $94 \mathrm{v}$.

36 Anselmi Gesta episcoporum Leodiensium, hg. von Rudolf KOEPKE u. a., in: MGH Scriptores (in Folio). Bd. 7: Chronica et gesta aevi Salici. Hannover 1846, S. 205 (II 29), dazu vgl. Pavel SPUNAR, La plus ancienne école en Bohême, in: Cahiers de la civilisation medievale 17 (1974), S. 125-128 und Marie BLÁHOVÁ, Pražské školy předuniversitního období, in: Documenta Pragensia 11 (1993), S. 27.

37 Vgl. u. a. Roman MICHAŁOWSKI, Święty Wojciech - biskup reformator w Europie X wieku, in: Zbigniew Dalewski (Hg.), Granica wschodnia cywilizacji zachodniej w sredniowieczu. Warszawa 2014, S. 204-210, oder mit dem Rücksicht an Überlieferung David KALHOUS, East meets West, West Meets East?: Constructing Difference in the First Life of St Adalbert and in the Life of St Neilos, in: Barbara Crostini - Ines Angeli Murzaku (Hgg.), Greek Monasticism in Southern Italy: the Life of St Neilos in Context. Routledge 2018, S. 282 ff. Für sein Bild im 11. Jahrhundert vgl. Petrus Damiani Gerhardum, apostolicae sedis episcopum electum, in: Die Briefe des Petrus Damiani. Bd. 2 (MGH Briefe der Deutschen Kaiserzeit. Bd. 4. Teil 2), hg. von Kurt REINDEL. München 1988, S. 168 (Nr. 57, ad a. 1058: „Quid beatum Adelbertum martyrem dicam? Qui nimirum, quoniam Boemiensis aecclesiae postposuit cathedram, monachum induens triumphalem martirii meruit invenire coronam? Utque ex industria de caeteris sileam, quidam sanctus paenitens, cuius me ad praesens vocabulum fugit, iam ante petennium fere episcopatu dimisso tandem angelo visitante praecipitur, ut ad episcopatum redeat..."). 
Tafel 1: Erste 12 Lagen der Handschrift Heiligenkreuz 217

\begin{tabular}{|c|c|c|c|c|c|c|}
\hline $\begin{array}{l}\text { Foliierung } \\
\text { (XIX. Jhdt.) }\end{array}$ & $\begin{array}{l}\text { Lagen- } \\
\text { reihe }\end{array}$ & $\begin{array}{l}\text { Lagen- } \\
\text { struktur }\end{array}$ & $\begin{array}{l}\text { Urspr. } \\
\text { Kustode } \\
\text { (X. Jhdt.) }\end{array}$ & $\begin{array}{l}\text { Jüngere } \\
\text { Kustode } \\
\text { (XII. Jhdt.) }\end{array}$ & \begin{tabular}{|l} 
Alte \\
Foliie- \\
rung \\
(XVII. \\
Jhdt.)
\end{tabular} & $\begin{array}{l}\text { Einträge des X./XI. Jhs. } \\
\text { Prov.: die Prager Kirche }\end{array}$ \\
\hline $1-6$ & 1. & III & & $\mathrm{I}^{9}$ & & $\begin{array}{l}\text { Fol. } 5^{\mathrm{v}}-6^{\mathrm{v}} \text { CDB I Nr. } 26 \text { (Sephan } \\
\text { V) }\end{array}$ \\
\hline 7-14 & 2. & IV & & $\mathrm{II}^{9}$ & & \\
\hline $15-22$ & 3. & IV & & III $^{9}$ & & \\
\hline $23-28$ & 4. & III & & IIII $^{9}$ & & \\
\hline $29-37$ & 5. & $\mathrm{IV}+1$ & & $\mathrm{~V}^{9}$ & $1-8$ & \\
\hline $38-44$ & 6. & $\mathrm{III}+1$ & & $\mathrm{VI}^{9}$ & $9-15$ & \\
\hline $45-52$ & 7. & IV & c & $\mathrm{VII}^{9}$ & $16-23$ & $\begin{array}{l}\text { Fol. } 51^{\mathrm{r}}-52^{\mathrm{v}} \text { Ammonitio } \\
\text { Postquam protoplasti }\end{array}$ \\
\hline $53-54$ & 8. & 2 & {$[\mathrm{~d}]$} & {$\left[\mathrm{VIII}^{9}\right]$} & $24-25$ & $\begin{array}{l}\text { Fol. } 53^{\mathrm{r}} \text { Ammonitio Postquam } \\
\text { protoplasti (Beendigung) } \\
\text { Ursprünglich: IV (Quaternio) }\end{array}$ \\
\hline $55-60$ & 9. & $\mathrm{II}+2$ & e & VIIII $^{9}$ & $26-31$ & Ursprünglich: IV (Quaternio) \\
\hline $61-68$ & 10. & IV & f & $\mathrm{X}^{9}$ & $32-39$ & \\
\hline $69-76$ & 11. & IV & {$[\mathrm{g}]$} & $\mathrm{XI}^{9}$ & $40-46$ & $\begin{array}{l}\text { Fol. } 76^{\mathrm{r}} \text { CDB I, Nr. } 37 \\
\text { Fol. } 42 \text { der alten Foliierung bis }\end{array}$ \\
\hline $77-81$ & 12. & $2 \times I+1$ & & $\mathrm{XII}^{9}$ & $47-51$ & $\begin{array}{l}\text { Fol. } 77^{\mathrm{r}}-77^{\mathrm{v}} \text { Sermo Quia semel } \\
\text { vestrę caritatis } \\
\text { Fol. } 78^{\mathrm{r}}-81^{\mathrm{r}} \text { CDB I, Nr. } 26 \\
\text { (Stephan V: Beendigung) }\end{array}$ \\
\hline
\end{tabular}

\section{LITERATURVERZEICHNIS}

\section{Quellen}

Codex diplomaticus et epistolarius Regni Bohemiae. Bd. 1, hg. von Gustav FRIEDRICH. Praha 1907.

Hinkmar von Rheims, Quae exsequi debeat episcopus, et qua cura tueri res et facultates ecclesiasticas, in: Jacques-Paul MIGNE (Hg.), Patrologia Latina. Bd. 125. Paris 1852, col. 1087-1094.

Raymond ETAIX, Un manuel de Pastorale de l'epoque carolingienne, in: Revue Benedictine 91 (1981), S. 105-133.

Benedict GSELL, Verzeichniss der Handschriften in der Bibliothek des Stiftes Heiligenkreuz (Handschriften-Verzeichnisse. Bd. 1). Wien 1891.

Das Homiliar des Bischofs von Prag (Beiträge zur Geschichte Böhmens, Abt. 1. Bd. 1), hg. von F. HECHT. Praha 1863.

Die Kapitulariensammlung des Ansegis (MGH Capitularia regum Francorum. Nova. Series. Bd. 1), hg. von Gerhard SCHMITZ. Hannover 1996, S. 71-191.

Leges Alamannorum (MGH Leges nationum Germanicarum. Bd. 5. Teil. 1.), hg. von Karl August ECKHARDT. Hannover 1966.

Magnae Moraviae Fontes Historici. Bd. 3, hg. von Dagmar BARTOŇKOVÁ - Lubomír HAVLÍK - Jaroslav LUDVÍKOVSKÝ - Radoslav VEČERKA. Brno 1969.

MGH Capitularia Episcoporum. Bd. 1. Hannover 1984. 
MGH LL Concilia. Bd. 2: Concilia aevi Karolini. Teil 1/1: 742-817. Hannover/Leipzig 1906. MGH LL Concilia. Bd. 4: Die Konzilien der karolingischen Teilreiche 860-874, hg. von Wilfried HARTMANN. Hannover 1998.

Monumenta Poloniae Historica. Series nova. Bd. 4. Teil 1, hg. von Jadwiga KARWASIŃSKA. Warszawa 1962.

Monumenta Poloniae Historica. Series nova. Bd. 4. Teil 2, hg. von Jadwiga KARWASIŃSKA. Warszawa 1969.

\section{Literatur}

Maximilian DIESENBERGER, Der Cvp 420 - die Gemeinschaft der Heiligen und ihre Gestaltung im frühmittelalterlichen Bayern, in: Monique Goullet - Martin Heinzelmann - Christiane Veyrard-Cosme (Hgg.), L'hagiographie merovingienne à travers ses reecritures. Ostfildern 2010, S. 219-248.

John ELDEVIK, Episcopal Power and Ecclesiastical Reform in the German Empire: Tithes, Lordship, and Community. Cambridge 2012.

Alois HAIDINGER- Franz LACKNER, Die Bibliothek und das Skriptorium des Stiftes Heiligenkreuz unter Abt Gottschalk (1134/1147) (Codices manuscripti et impressi. Zeitschrift für Buchgeschichte - Supplementum 11). Purkersdorf 2015.

Silke HANSEN, Rechtscorpora in den Handschriften CLM 3853, Heiligenkreuz 217 und Par. Lat. 3878. Tübingen 1992 (ungedruckte Magisterarbeit).

Wilfried HARTMANN, Kirche und Kirchenrecht um 900: die Bedeutung der spätkarolingischen Zeit für Tradition und Innovation im kirchlichen Recht. Hannover 2008.

Wilfried HARTMANN, Das Konzil von Worms 868: Überlieferung und Bedeutung. Göttingen 1977.

Dalibor HAVEL, Počátky latinské písemné kultury v českých zemích: nejstarši latinské rukopisy a zlomky $v$ Čechách a na Moravě. Brno 2018.

Mayke de JONG, To the Limits of Kinship: Anti-Incest Legislation in the Early Medieval West (500-900), in: Jan BREMMMER (Hg.), From Sappho to de Sade: Moments in the History of Sexuality. London 1989, S. 36-59.

Lotte KÉRY, Canonical collections of the Early Middle Ages, ca. 400-1140: a Biographical Guide to the Manuscripts and Literature. Washington, DC 1999.

Raymund KOTTJE, Die Bussbücher Halitgars von Cambrai und des Hrabanus Maurus: ihre Überlieferung und ihre Quellen. Berlin/New York 1980.

Gert MELVILLE, Spätmittelalterliche Geschichtskompendien: eine Aufgabenstellung, in: Römische historische Mitteilungen 22 (1980), S. 51-104.

Hubert MORDEK, Bibliotheca capitularium regum Francorum manuscripta: Überlieferung und Traditionszusammenhang der fränkischen Herrschererlasse. München 1995.

Rostislav NOVÝ - Jiř́ SLÁMA, Slavníkovci ve středověkém písemnictví. Praha 1987.

Rudolf POKORNY, Ein unbekannter Synodalsermo Arns von Salzburg, in: Deutsches Archiv 39 (1983), S. 379-394.

Petr SOMMER, Smrt kněžny Ludmily a začátky české sakrální architektury, in: ČČH 98 (2000), S. 229-260.

Petr SOMMER, Začátky křstanství v Čechách: kapitoly $z$ dějin raně středověké duchovní kultury. Praha 2001.

Martina STRATMANN, Hinkmar von Reims als Verwalter von Bistum und Kirchenprovinz. Sigmaringen 1991.

Wilhelm WATTENBACH, Beitrage zur Geschichte der christlichen Kirche in Maehren und Boehmen. Wien 1849.

Wilhelm WATTENBACH, Reise nach Österreich in den Jahren 1847, 1848, 1849, in: Archiv der Gesellschaft für altere deutsche Geschichtskunde 10 (1851), S. S. 426-693.

Franz ZAGIBA, Der Kodex 217 der Stiftsbibliothek Heiligenkreuz in Niederosterreich als Quelle zur Kirchengeschichte Böhmens und Mährens, in: Millenium dioeceseos Pragensis 973-1973: Beiträge zur Kirchengeschichte Mitteleuropas im 9.-11. Jahrhundert. Wien/Köln/Graz 1974, S. 64-72.

Jana ZACHOVÁ - Dušan TŘEŠTÍK, Adhortace De ammonicione ad presbiteros a biskup Vojtěch, in: ČC̆H 99 (2001), S. 279-293. 